\title{
Gradient, Texture Driven Based Dynamic- Histogram Equalization For Medical Image Enhancement
}

\author{
Vidyasaraswathi $\mathrm{H} \mathrm{N}^{1 *}$, Hanumantharaju $\mathrm{M} \mathrm{C}^{2}$ \\ ${ }^{1}$ Dept. of ECE, Bangalore Institute of Technology, Karnataka, India \\ ${ }^{2}$ Dept. of ECE, BMS Institute of Technology \& Management, Karnataka, India \\ Email: vidya.gowdaec@gmail.com
}

Received: January 23, 2021. Revised: June 30, 2021. Accepted: July 21, 2021. Published: July 22, 2021.

\begin{abstract}
In many clinical diagnostic measurements, medical images play some significant role but often suffer from various types of noise and low-luminance, which causes some notable changes in overall system accuracy with misdiagnosis rate. To improve the visual appearance of object regions in medical images, image enhancement techniques are used as potential pre-processing techniques. Due to its simplicity and easiness of implementation, histogram equalization is widely preferred in many applications. But due to its mapping function based image transformation during enhancement process affect the biomedical patterns which are essential for diagnosis. To mitigate these issues in medical images, a new method based on gradient computations and Texture Driven based Dynamic histogram equalization (GTDDHE) is accomplished to increase the visual perception. The spatial texture pattern is also included to ensure the texture retention and associated control over its variations during histogram modifications. Experimental results on MRI, CT images, eyes images from medical image datasets and quantitative analysis by PSNR, structural similarity index measurement (SSIM), information entropy (IE) and validated that the proposed method offers improved quality with maximum retention of biomedical patterns across all types of medical images.
\end{abstract}

Keywords: Gradient, Local binary pattern, Image enhancement etc.

\section{INTRODUCTION}

Image enhancement is one of the essential methodology used widely in many digital image processing system, has been an active area of research in recent years due to the invention of vision based applications. Most of the existing image enhancement studies developed some spatial transformations to improve the quality of the image and reducing the noise presence for better visualization since the visibility level is poor in any low-contrast image features.

In recent, imaging processing is steadily emerging in medical fields for several diagnostic measurements and plays significant role in examination of the patient health condition. Different type's medical images are used for various clinical diagnoses. In general medical images are comprise of several complicated regions, which required some unique pre processing models to simplify the complications that arises in various stages of CAD system. Moreover medial images are not carrying fine details for an accurate classification due to poor lighting conditions, external noise interferences and other restrictions related to imaging devices which makes the clinical diagnosis a difficult task to accomplish. Medical images itself comes in different format namely magnetic resonance imaging (MRI), chest X-ray (CXR), computerized tomography (CT) and mammographic images etc. During classification only the pixel intensity differences among various region and object boundary information are playing vital role. 
Therefore, for improved classification rate in medical images both quality and contrast need to improve collectively in such way that each object can be easily observed during analyses.

Though the core objective of any image enhancement technique is to improve quality while suppressing noise, it is essential to restore the basic details without causing any significant changes. Even in some cases image restoration is also considered as image enhancement; but still it is only deals with appropriate estimation of the actual image from observed one. Most of the existing image-enhancement techniques not consider degradation process, in general, it is not modelled; however, for medical images degradation process may causes some notable performance loss with the inclusion of enhancement techniques.

Among various methods Histogram based techniques for image enhancement is widely investigated in many works which is based on equalization of the histogram of the values. Histogram Equalization (HE) has some inherent characteristics of changing the intensity values and spatial patterns which cause some notable problems in medical imaging. To overcome this issue, Contrast limited adaptive HE (CLAHE) is proposed as potential model for medical image analysis. Shome et al. (2011) [1] proves that contrast limited image enhancement using histogram equalization explores the abnormalities with improved quality.

In general the cumulative effect of image enhancement process in medical image spatial patterns and its influence in clinical diagnostic accuracy is investigated in many existing works. This work presents the gradient and texture driven dynamic sub-histogram equalization technique based on statistical measurements of various image attributes such as intensity bound, texture driven spatial correlations and gradient driven object orientations etc.

Several existing works [2-3] well proved that the texture retention and boundary details are essential measures which play significant role in clinical diagnostics measurements [4]. In this paper, intensity, gradient and texture measures are used for contrast limited adaptive non linear histogram equalization which has metrics as follows:

- The spatial orientations of object pixels are remain unaltered while visual appearance is improved.
- The statistical significance of texture information is considered and its retention is ensured in enhanced image.

\section{RELATED WORKS}

Bhateja et al. (2013) [5] introduced non-linear framework which employs a robust polynomial filter for enhancing the mammographic abnormalities. Through filtering approach mammographic masses are improved irrespective to the background tissues. The experimental results prove that contrast is increased with significant suppression of noise level and preserved edges without causing any notable blurring artifacts. Singh et al. (2014) [6] proposed exposure based Sub-Image Histogram Equalization (ESIHE) to improve the enhancement of low exposure image. Here by dividing the input image into sub-images using pre defined thresholds and intensity levels. To control enhancement rate the histogram is clipped using a threshold value and gray level occurrences.

colour image enhancement technique proposed by Wong et al. (2016) [7] increase the colour information and suppressing the artefacts. Here equalization is performed recursively to extend the intensity ranges optimally using the golden section search algorithm. The histogram values are equalized independently over sub images. Color image enhancement developed by Lee et al. (2017) [8] carried out intensity HE based on hue and saturation values in HSI color space. As compared to conventional HE the visual colourfulness is well preserved in color space conversion driven enhancement process. The performance metrics are validated using both back-lighting and night-time image sets are used for experimentation. Wan et al. (2018) [9] introduced histogram partition and brightness correction for infrared image enhancement technique. Here based on of human visual system visual correction factor are introduced and grayscale re-mapping process is carried out to suppress the noise level. In addition to this, brightness correction is also accomplished using particle swarm optimization algorithm.

Histogram shifting based contrast enhancement is developed in Ying et al. (2019) [10] for reversible data hiding with minimal distortions over embedded image. Here transfer matrix is constructed by maximizing the entropy of the histogram and embedding is performed accordingly to increase the embedding capacity. Srinivas et al. (2020) [11] 
combines the contrast and Color enhancement using spatial contextual similarity histogram model. Based on the deviation of the pixel values in luminance channel cumulative distribution function is formulated and contrast-enhancement is achieved through intensity transformation. And chromatic information is used for adaptive color restoration to restore the consistent color information's. The hierarchical equalization model developed by Bai et al. (2020) [12] comprises of four stages namely centre regionalization, local equalization, global equalization and multi-scale fusion. Here equalization strategy is used to perform both for both contrast improvements and correct the color characteristics of the each channel. Kandhway et al. (2020) [13] proposed highly optimized contrast and edge enhancement methodologies for bio medical images to explore the different anatomical characteristic of the diseases. Here plateau limit is used to select the histogram based on min, max, mean, and median of it with some adjustable parameter. Based on relative vacancy of histogram bins the residue blocks are generated and metaheuristic algorithm is incorporated based on a fitness function which includes two functions with edge, entropy, gray level co-occurrence matrix (GLCM) for contrast, and energy for visual impairments.

Gamma correction based dynamic clipped HE proposed by Subramani et al. (2020) [14] control the color enhancement rate using mean values. Here both clipping and gamma correction is performed over decomposed image sub blocks to preserves basic image attributes like entropy, colors, saturation etc. the same histogram clipping operations proposed in Bhandari et al. (2020) [15] used salp swarm algorithm (SSA) optimization to formulate the optimal plateau limit (PLs) to overcome the performance degradations caused by illuminations and backlighting effect and suppress the associated artifacts.

In all above discussed models for contrast enhancement cause some undesirable effects which will reduce the diagnostic measurements of the biomedical images. Histogram based image enhancements have some limitations like performance trade off over texture retention, boundary diffusion and artifacts related problems etc. Moreover enhancement from conventional methods required some statistical characteristics of the input images which are not available for most biomedical image modalities. Therefore it is essential to narrow down the information loss and parametric requirements of existing $\mathrm{HE}$ techniques without compromising the reconstruction quality. In this work image attributes driven histogram equalization is proposed which includes various image characteristics namely luminations, gradient and texture information. Here the trade off in the SSIM texture measure is narrow down and the penalty gap due to over enhancement is also suppressed during contrast enhancement. Here equalization is processed over luminance channel and texture and object boundary details are preserved using threshold bound computed in identical channels to accomplish contrast limited image enhancement: the proposed GTDDHE approach can improves the contrast and explore precise biomedical abnormalities in each medical images.

\section{PROPOSED METHOD}

The image enhancement through GTDDHE method modifies both the dynamic range and contrast levels to formulate the histogram in some desired shape using some mapping function as cumulative distribution function. This stretching of histogram peak values directly relates the intensity level changes and contrast is adjusted accordingly as shown in Figure 1.

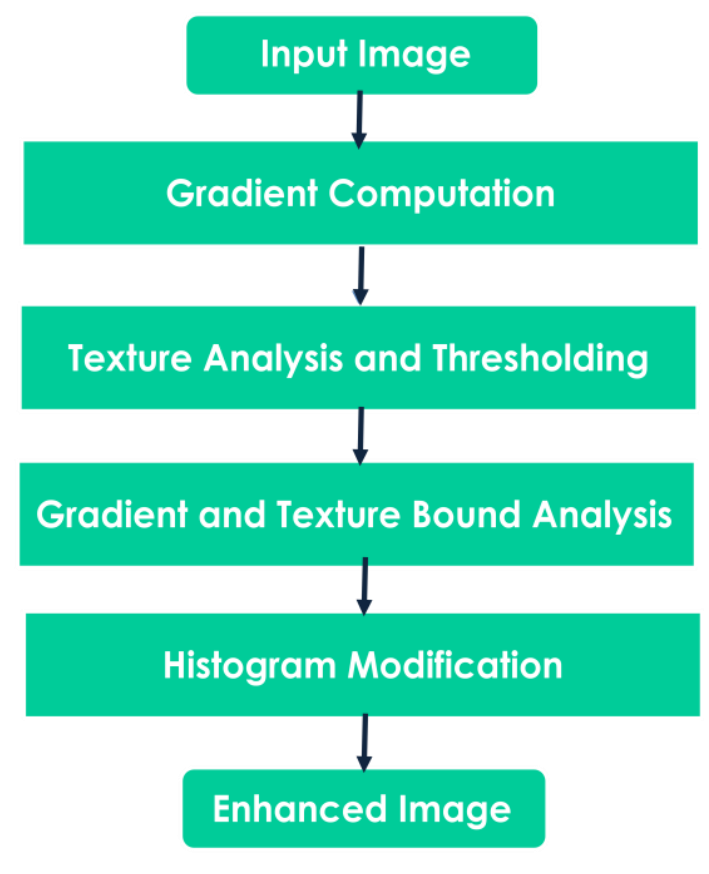

Figure.1 Proposed GTDDHE method flow graph.

\section{A. Gradient Approach}

Gradients of pixels are used to express the cooccurrence variations of pixels inside every region. The computation of local pixel co-occurrence in the similar region discloses the correlative nature of the 
neighbouring pixels precisely. The gradient measure in each object model contributes in identifying the magnitude shift between the complexes and smooth region. Here, a significant change in a gradient indicates object discriminations. This simple linear variation is most effective for modelling the object of interest. The gradient with the variance measure is shown in Figure 2(a).
Figure.2 (a) Gradient

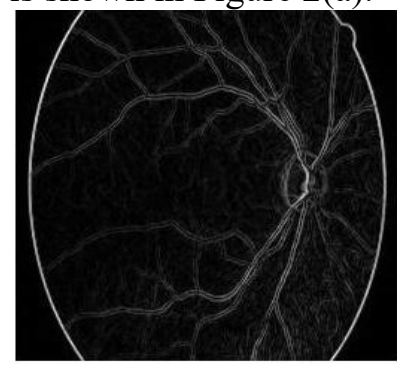

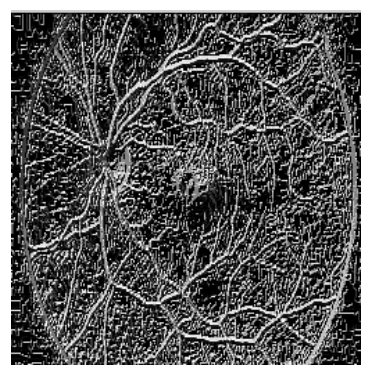

Figure.2 (b) Texture Output

\section{B. Spatial invariant Texture Model}

Texture models are used to decide which region contains the region of interest in medical objects. For texture analysis in vision applications, the local binary pattern (LBP) operator has been used as a standard feature-based approach. Even small changes in pixel intensity values (due to illumination or dynamic changes) give a changed LBP code. Also invariant and uniform textures are efficient in opposition to any dynamic changes. The vital mathematical explanation of the LBP operation is described in the accompanying equations below.

$$
\begin{gathered}
C=\sum_{d=0}^{7} 2^{d} * B_{d} \\
B_{d} \text { for } 0=0: \sum_{d=0}^{7} t d>c, d \in[0,7] \\
B_{d} \text { for } 1=1: \sum_{d=0}^{7} t d<c, d \in[0,7]
\end{gathered}
$$

Generally, the binary pattern is termed uniform in case the LBP binary pattern comprises of two bitwise transitions from 1 to 0 or 0 to 1 atleast and have circular binary pattern. For static textures, rotation-invariant features of this kind are not dependent of input image angle as shown in Figure 2(b). Dynamic textures (DT) and uniform textures, combined with rotational-invariant feature description, is the key element where local features obtain the ability to describe motion and provide the most discriminative patterns for object modelling.

\section{Detailed Steps of Proposed Method}

Here input histogram values are decomposed into several groups based on threshold bounds and analyzes its variations with gradient and texture bounds for equalization process as given below:

//Input - Low contrast Image

//Output - Enhanced image

Step 1: Set sigma $(\sigma) \quad$ //variance value

Step 2: Extract gradient channel using Gaussian derivative - CG channel

$$
\text { Gauss }=\frac{1}{\sqrt{2 \pi \sigma}} e^{x^{2} /\left(-2 \sigma^{2}\right)}
$$

Step 3: Extract Texture channel using LBP -

$T$ channel

$\mathrm{LBP}=2^{7} \mathrm{p} 0+2^{6} \mathrm{p} 1+2^{5} \mathrm{p} 2+2^{4} \mathrm{p} 3+2^{3} \mathrm{p} 4+2^{2} \mathrm{p} 5+2^{1} \mathrm{p} 6+2^{0} \mathrm{p} 7$

Where, $\mathrm{p} 0-\mathrm{p} 7$ true value comparison of centre pixels with 8 surrounding pixels in $3 \times 3$ macro block

Step 4: Extract $3 \times 3$ overlapping window

Let $\mathrm{P}_{\mathrm{ij}}$ - current pixel as well as center pixel in $3 \times 3$ macro block

$\mathrm{CG}_{\mathrm{ij}}$ - current pixel gradient value

$\mathrm{T}_{\mathrm{ij}}$ - current pixel texture bound value

$$
\begin{array}{r}
\text { if }\left(\mathrm{P}_{\mathrm{ij}}>\mathrm{T}_{\text {lower }}\right) \& \&\left(\mathrm{P}_{\mathrm{ij}}>\mathrm{T}_{\text {upper }}\right) \& \&\left(\mathrm{CG}_{\mathrm{ij}}<\mathrm{P}_{\mathrm{ij}}\right) \\
\mathrm{OR}\left(1, \mathrm{~T}_{\mathrm{gr}}\right)+1 \quad / / \text { Spatial Orientation } \\
\text { measure for boundary preservations }
\end{array}
$$

end

Step 5: Apply histogram equalization over each sub groups of $\mathrm{P}_{\text {out }}$

$$
\begin{aligned}
& \text { if }\left(\mathrm{T}_{\mathrm{ij}}>\mathrm{T}_{\text {lower }}\right) \& \&\left(\mathrm{~T}_{\mathrm{ij}}<\mathrm{T}_{\text {upper }}\right) \\
& \mathrm{P}_{\text {out }}=\left(2^{\mathrm{L}}-1\right) \quad \mathrm{x} \quad \mathrm{OR}\left(1, \mathrm{~T}_{\mathrm{gr}}\right) / 2^{\mathrm{M}} \times 2^{\mathrm{N}} \quad / / \text { Texture } \\
& \text { Preservation }
\end{aligned}
$$

end

Where,

$\mathrm{T}_{\text {lower, }} \mathrm{T}_{\text {upper }}-$ Histogram threshold bounds for groups.

$\mathrm{T}_{\mathrm{gr}-\text { number of histogram groups. }}$

L- histogram levels

$\mathrm{M}, \mathrm{N}$ - size of input images 


\section{EXPERIMENTAL RESULTS}

The performance metric evaluation of the GTDDHE method is experimented over the different biomedical dataset which includes eye images, MRI and $\mathrm{CT}$ images which are collected from various sources. From each dataset 30 images of different level of textures and complication in patterns. Performance metric evaluation of the proposed GTDDHE is experimented using different quantitative parameters.

The peak signal-to-noise ratio (PSNR) measure is used to validate the reconstruction quality of proposed image restoration through $\mathrm{SR}$ as given below:

$$
\operatorname{PSNR}(d B)=10 \log _{10}\left[\frac{\left(2^{n}-1\right)^{2}}{M S E}\right]
$$

Where MSE denotes the mean squared error.

The structural similarity index (SSIM) is used to measure the structural similarity between input LR and HR images based on independent parametric comparisons such as luminance $(\mu)$, contrast $(\sigma)$ as follows:

$\operatorname{SSIM}(e, f)=\frac{\left(2 \mu_{\mathrm{e}} \mu_{\mathrm{f}}+\mathrm{C}_{1}\right)\left(2 \sigma_{\mathrm{ef}}+\mathrm{C}_{2}\right)}{\left(\mu_{\mathrm{e}}^{2}+\mu_{\mathrm{f}}^{2}+\mathrm{C}_{1}\right)\left(\sigma_{\mathrm{e}}^{2}+\sigma_{\mathrm{f}}^{2}+\mathrm{C}_{2}\right)}$

And the measure the structural similarity is evaluated based on its correlations.

Entropy measures the highness of information in enhanced image as shown in Eqn. (7) and its improvement is measured using Measure of enhancement by entropy (EMEE) value as shown in Eqn. (8)

$$
\text { Entropy }(E)=\sum_{x, y}^{255} \operatorname{Pr}(b, c)(-\ln \operatorname{Pr}(b, c))
$$

where $\operatorname{Pr}(b, c)$ denotes the probability of the difference between two successive pixels $\mathrm{b}$ and $\mathrm{c}$.

$E M E E=\frac{1}{N} \sum \frac{I_{\max }}{I_{\min }} 20 \log \frac{I_{\max }}{I_{\min }}$

Average gradient (AG) measure is used to explore the relative clarity of the enhanced output image using Eqn. (9)

$$
A G=\frac{1}{(M-1)(N-1)} \sum_{x} \sum_{y} \frac{|G(b, c)|}{\sqrt{2}}
$$

Where $|\mathrm{G}(\mathrm{b}, \mathrm{c})|$ denotes absolute value of the Magnitude of Gradient.

Finally Contrast improvement index (CII) is calculated to quantify contrast enhancement of an output image using Eqn. (10)

$$
C I I=\frac{C_{\text {input }}}{C_{\text {output }}}
$$

Where $\mathrm{C}_{\text {output }}$ and $\mathrm{C}_{\text {input }}$ denote the enhanced and input images contrast values respectively.

\section{A. Performance Analysis}

Simulation outcome of the GTDDHE approach and the four other methods are shown in figure. 3 and they are investigated both quantitatively and qualitatively. The proposed GTDDHE offers highly normalized histogram distribution as shown in Figure 4.

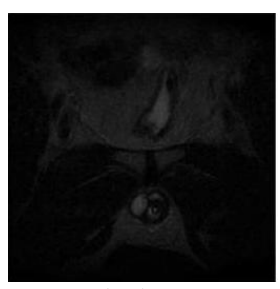

(a1)

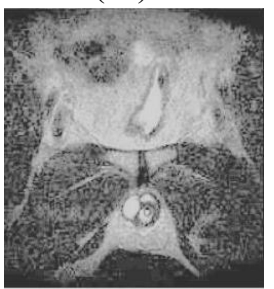

(b1)

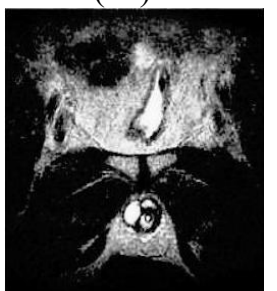

(c1)

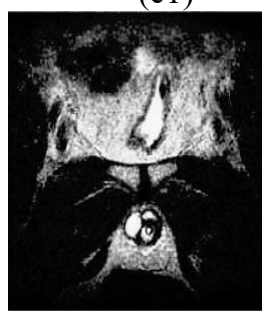

(d1)

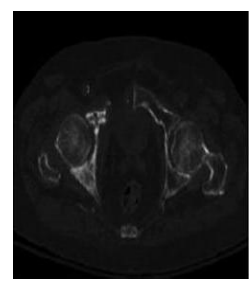

(a2)

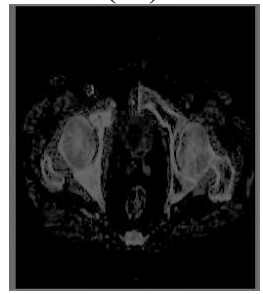

(b2)

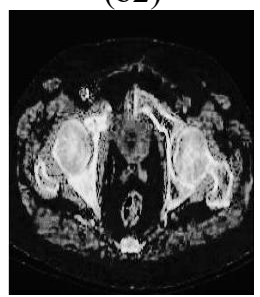

(c2)

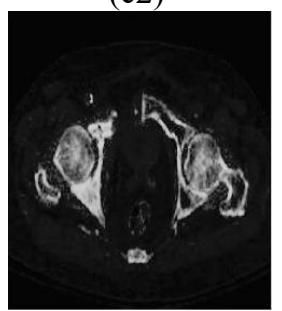

(d2)

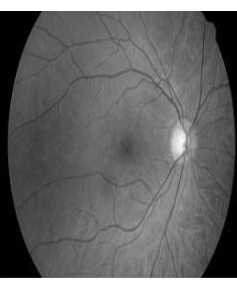

(a3)

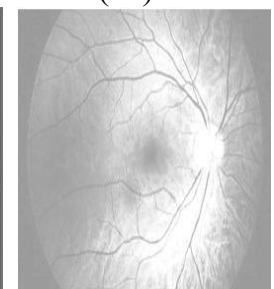

(b3)

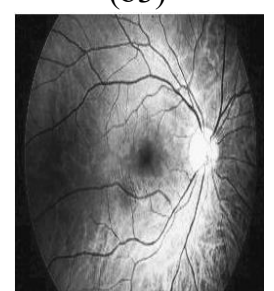

(c3)

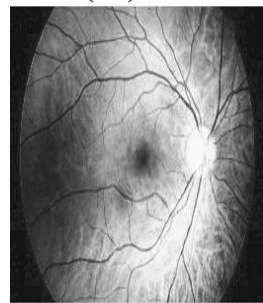

(d3) 


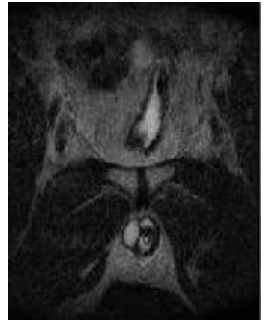

(e1)

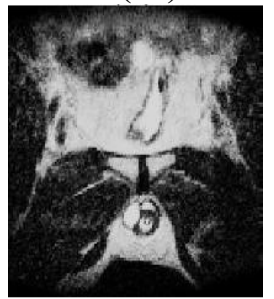

(f1)

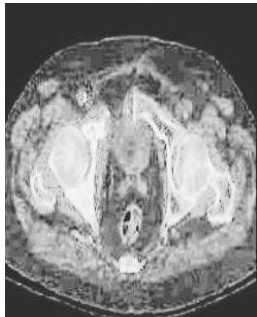

(e2)

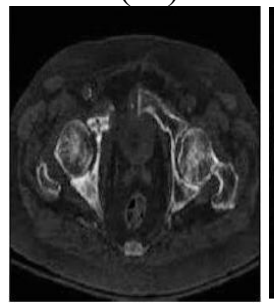

(f2)

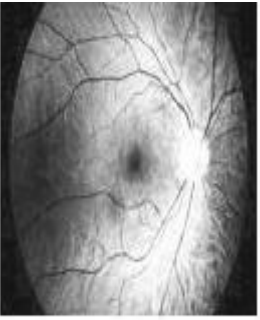

(e3)

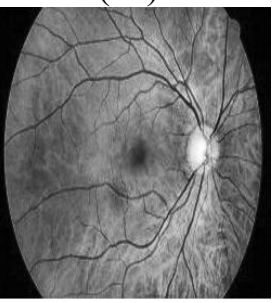

(f3)
Figure.3 Qualitative valuation of low-contrast image enhancement: (a1-a3) Original low contrast images, (b1b3) enhanced output image using HE [18], (c1-c3) enhanced output image using DSIHE [19], (d1-d3) enhanced output image based on RMSHE [20], (e1-e3) enhanced output image based on CLAHE [21], and (f1f3) enhanced output image based on proposed GTDDHE method.

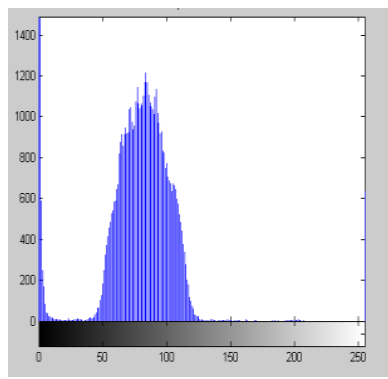

(a)Input Histogram

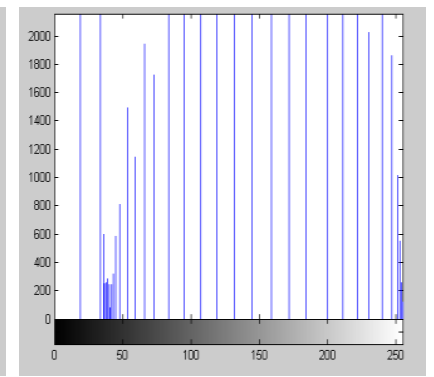

(b) Output Histogram
Figure.4. Histogram comparison plot.

The proposed GTDDHE offers peak PSNR over all classes of given image sets as shows in table.1. It is also proves that inclusion of texture interpolation and gradient measures increase the PSNR and SSIM measure with improved spatial details. The proposed method outperformance both in terms of quality as well as spatial and boundary information retention. As shown in figure.5 and figure.6, the GTDDHE method shows better results compared to DWTSVD[17], HE[18], DSIHE[19], RMSHE[20] AND CLAHE[21].

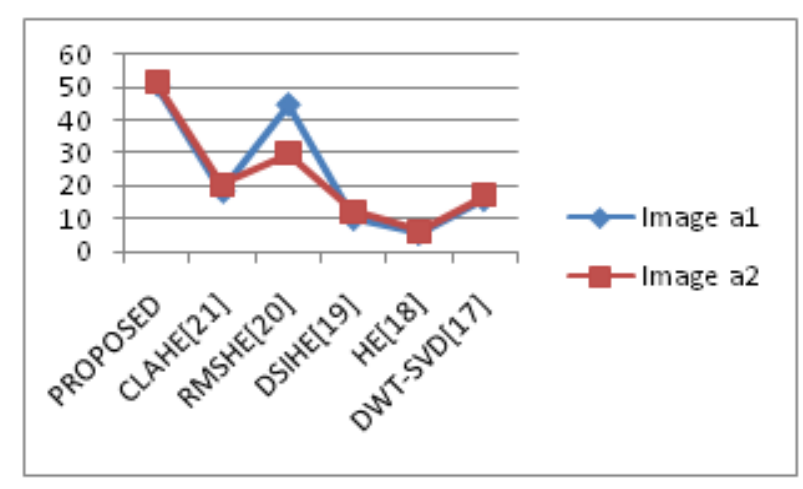

Figure.5 Comparison of PSNR

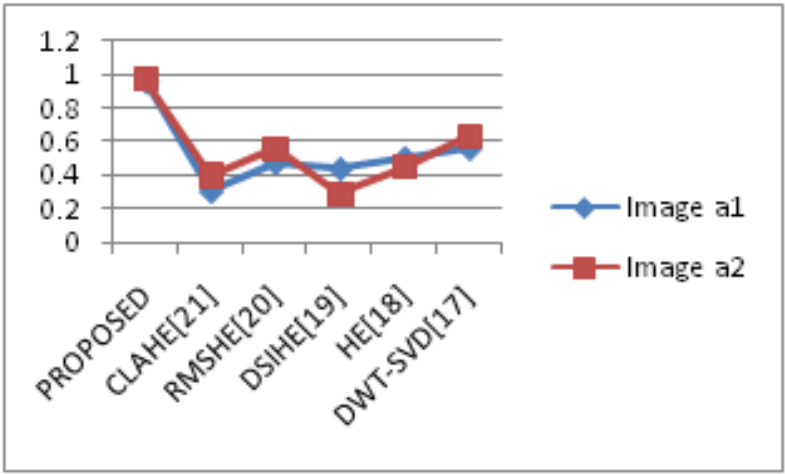

Figure.6 Comparison of SSIM

Table 1. Average metric values for 60 images

\begin{tabular}{|c|c|c|c|c|c|}
\hline $\begin{array}{c}\text { Methods/ } \\
\text { Measures }\end{array}$ & HE & $\begin{array}{c}\text { DSIH } \\
\mathbf{E}\end{array}$ & $\begin{array}{c}\text { RMSH } \\
\mathbf{E}\end{array}$ & $\begin{array}{c}\text { CLAH } \\
\mathbf{E}\end{array}$ & $\begin{array}{c}\text { GTDD } \\
\text { HE }\end{array}$ \\
\hline PSNR & 12.90 & 17.230 & 29.724 & 19.365 & 51.345 \\
\hline SSIM & 0.475 & 0.6185 & 0.6206 & 0.5666 & 0.9905 \\
\hline MSE & 3333 & 1060.8 & 500.553 & 859.58 & 0.0680 \\
\hline $\begin{array}{c}\text { ENTRO } \\
\text { PY }\end{array}$ & 4.967 & 5.6764 & 5.9957 & 6.0665 & 6.72 \\
\hline EMME & 0.0414 & 0.0583 & 0.07407 & 0.0801 & 0.0684 \\
\hline MC & 0.42 & 0.67 & 0.81 & 0.79 & 1.0415 \\
\hline CII & 0.6162 & 0.7578 & 0.87419 & 1.2196 & 1.96205 \\
\hline AG & 50.184 & 45.749 & 48.4786 & 54.296 & 61.2167 \\
\hline
\end{tabular}




\section{CONCLUSION}

This paper investigates the quantitative effect of histogram equalization based image enhancement on various medical image modalities. The proposed color space conversion-based image enhancement technique shows significant quality improvements and overcome the distortions caused by over enhancement and associated problems in pattern classification. Here spatial texture pattern and gradient threshold bounds are applied not only for improving visual appearance of enhanced output image, but also for significantly restore the basic details required for accurate medical pattern analyzes.

\section{REFERENCES}

[1]Shome, Saikat Kumar, and Siva Ram Krishna Vadali, "Enhancement of diabetic retinopathy imagery using contrast limited adaptive histogram equalization", International Journal of Computer Science and Information Technologies, Vol.2, No. 6, pp.2694-2699, 2011.

[2]Huang, Yu-Len, Kao-Lun Wang, and Dar-Ren Chen, "Diagnosis of breast tumors with ultrasonic texture analysis using support vector machines", Neural Computing \& Applications, Vol.15, No. 2, pp.164-169, 2006.

[3]El-Gamal, Fatma El-Zahraa A., Mohammed M. Elmogy, Mohammed Ghazal, Ahmed Atwan, Gregory N. Barnes, Manuel F. Casanova, Robert Keynton, and Ayman S. El-Baz, "A novel CAD system for local and global early diagnosis of Alzheimer's disease based on PIB-PET scans", IEEE International Conference on Image Processing (ICIP), pp. 3270-3274, 2017.

[4]Qi, Qunfen, Paul J. Scott, Xiangqian Jiang, and Wenlong Lu, "Design and implementation of an integrated surface texture information system for design, manufacture and measurement", ComputerAided Design, Vol. 57, pp.41-53, 2014.

[5]Bhateja, Vikrant, Mukul Misra, Shabana Urooj, and Aime Lay-Ekuakille, "A robust polynomial filtering framework for mammographic image enhancement from biomedical sensors", IEEE Sensors Journal, Vol.13, No.11, pp.4147-4156, 2013.

[6]Singh, Kuldeep, and Rajiv Kapoor, "Image enhancement using exposure based sub image histogram equalization", Pattern Recognition Letters, Vol.36, pp.10-14,2014.
[7]Wong, Chin Yeow, Shilong Liu, San Chi Liu, Md Arifur Rahman, Stephen Ching-Feng Lin, Guannan Jiang, Ngaiming Kwok, and Haiyan Shi, "Image contrast enhancement using histogram equalization with maximum intensity coverage", Journal of Modern Optics, Vol.63, No.16,pp.1618-1629,2016.

[8]Lee, Su-Ling, and Chien-Cheng Tseng, "Color image enhancement using histogram equalization method without changing hue and saturation", IEEE International Conference on Consumer ElectronicsTaiwan (ICCE-TW), pp. 305-306, 2017.

[9]Wan, Minjie, Guohua Gu, Weixian Qian, Kan Ren, Qian Chen, and Xavier Maldague, "Infrared image enhancement using adaptive histogram partition and brightness correction", Remote Sensing, No. 10,682, 2018.

[10]Ying, Qichao, Zhenxing Qian, Xinpeng Zhang, and Dengpan Ye, "Reversible data hiding with image enhancement using histogram shifting", IEEE Access, pp.46506-46521, 2019.

[11]Srinivas, Kankanala, Ashish Kumar Bhandari, and Anurag Singh. "Low-contrast image enhancement using spatial contextual similarity histogram computation and color reconstruction." Journal of the Franklin Institute, Vol.357, No. 18, pp.13941-13963, 2020.

[12] Bai, Linfeng, Weidong Zhang, Xipeng Pan, and Chenping Zhao, "Underwater image enhancement based on global and local equalization of histogram and dual-image multi-scale fusion", IEEE Access, pp. 128973-128990,2020.

[13]Kandhway, Pankaj, Ashish Kumar Bhandari, and Anurag Singh, "A novel reformed histogram equalization based medical image contrast enhancement using krill herd optimization", Biomedical Signal Processing and Control, pp.101677, 2020.

[14]Subramani, Bharath, and Magudeeswaran Veluchamy, "Quadrant dynamic clipped histogram equalization with gamma correction for color image enhancement", Color Research \& Application, 2020.

[15] Bhandari, Ashish Kumar, Pankaj Kandhway, and Shubham Maurya", Salp swarm algorithm based optimally weighted histogram framework for image enhancement", IEEE Transactions on Instrumentation and Measurement, 2020. 
[16]Subramani, Bharath, and Magudeeswaran Veluchamy. "Fuzzy contextual inference system for medical image enhancement", Measurement,148 pp.106967-106976, 2019.

[17]Fathi Kallel, Ahmed Ben Hamida,"A new adaptive gamma correction-based algorithm using DWT-SVD for non-contrast CT image enhancement", IEEE Trans. Nano Biosci, Vol.16, No.8, pp.666-675, 2017.

[18]Gonzalez RC, Woods RE, Eddins SL Digital image processing using MATLAB Gatesmark Publishing, USA, 2009.

[19]Yu Wang, Qian Chen, Baomin Zhang, "Image Enhancement Based On Equal Area Dualistic SubImage Histogram Equalization Method", IEEE Transactions on Consumer Electronics, Vol. 45, No. 1,pp.68-75, 1999.

[20]Soong-Der Chen, Abd. Rahman Ramli, "Contrast Enhancement using Recursive MeanSeparate Histogram Equalization for Scalable Brightness Preservation", IEEE, pp.1301-1309, 2003.

[21] Himanshu Singh, Vivek Singh, "A Comparative Analysis on Histogram Equalization Techniques for Medical Image Enhancement", International Journals of Advanced Research in Computer Science and Software Engineering, Vol.7, No.6, pp.364-370,2017.

\section{Creative Commons Attribution License 4.0 (Attribution 4.0 International, CC BY 4.0)}

This article is published under the terms of the Creative Commons Attribution License 4.0

https://creativecommons.org/licenses/by/4.0/deed.en_US 